\title{
Surface Distresses Detection of Pavement Based on Digital Image Processing
}

\author{
Aiguo Ouyang ${ }^{1}$, Chagen Luo ${ }^{1}$, and Chao Zhou ${ }^{2}$ \\ ${ }^{1}$ Key Laboratory of Conveyance and Equipment, Ministry of Education, \\ East China Jiaotong University, Nanchang, China \\ ${ }^{2}$ Jiangxi Agricultural University, Nanchang, China
}

\begin{abstract}
Pavement crack is the main form of early diseases of pavement. The use of digital photography to record pavement images and subsequent crack detection and classification has undergone continuous improvements over the past decade. Digital image processing has been applied to detect the pavement crack for its advantages of large amount of information and automatic detection. The applications of digital image processing in pavement crack detection, distresses classification and evaluation were reviewed in the paper. The key problems were analyzed, such as image enhancement, image segmentation and edge detection. The experiment results of the commonly used algorithms forcefully supported following conclusion: the noise in pavement crack images is effectively removed by median filtering, the histogram modification technique is a useable segmentation approach, the canny edge detection is an ideal identification approach of pavement distresses.
\end{abstract}

Keywords: Digital Image processing, Crack detection, Edge detection, Image segmentation.

\section{Introduction}

Maintenance of pavements is an important aspect for the Departments of Transportation all over the world. The first step towards maintenance is the identification of pavement distresses and their documentation for further action. Pavement distresses are visible imperfections on the surface of the pavements. Accurate evaluations will result in a better chance that resources will be distributed normally. Thus, yield a better service condition [1]. Pavement could be evaluated through the different types of distress experienced, such as cracking, disintegration and surface deformation. At present, there were various methods for conducting distress surveys, recording and analysing distress survey data [2]. Pavement engineers had long recognized the importance of distress information in quantifying the quality of pavements. Traditionally, pavement condition data are gathered by human inspectors who walk or drive along the road to assess the distresses and subsequently produce report sheets, but it is high cost and time consuming. Worse still, work has to be done along fast moving traffic. Such condition would endanger the safety of the personnel involved. Finally, large differences will 
exist between the actual condition and evaluation results because of the subjectivity of the evaluation process.

In the wake of tedious manual measurements and safety issues, a variety of types of methods have been devised to identify the cracks on pavements apart from the crude process of manual inspection. Image processing, ultrasonic detection and infrared detection, the most widely reported of the automated methods is that known as WiseCrax. Wisecrax [3] is an example of a commercially available device that uses infrared imaging to detect cracks on pavements. A camera is mounted on a vehicle with that takes pictures of the pavements continuously. Images are processed off-line overnight at the office workstation by a unique open architecture process using advanced image recognition software. Typical survey vehicle configuration consists of one or more downward-facing video cameras, at least one forward facing camera for perspective, and any number of additional cameras for the capture of right-of-way, shoulder, signage, and other information depending on agency requirements (as shown in Figure 1).

New methods are being devised to identify cracks more efficiently and get closer towards perfection as good as the human eye and are still in the process. Ye et al. [4] presented a crack width detection method to extract the crack image of pavement distresses and calculate the crack width. Li et al. [5] proposed a pavement crack image analysis approach based on the image dodging to improve the reliability of the pavement crack recognition. Existing automatic real-time detection systems focus on the low identification rate and classification difficulty [6]. First, a lot of noise brings in pavement images caused by the road environment itself. Second, it is lack of easy and effective identification and classification algorithm [7]. Although some auto-inspection

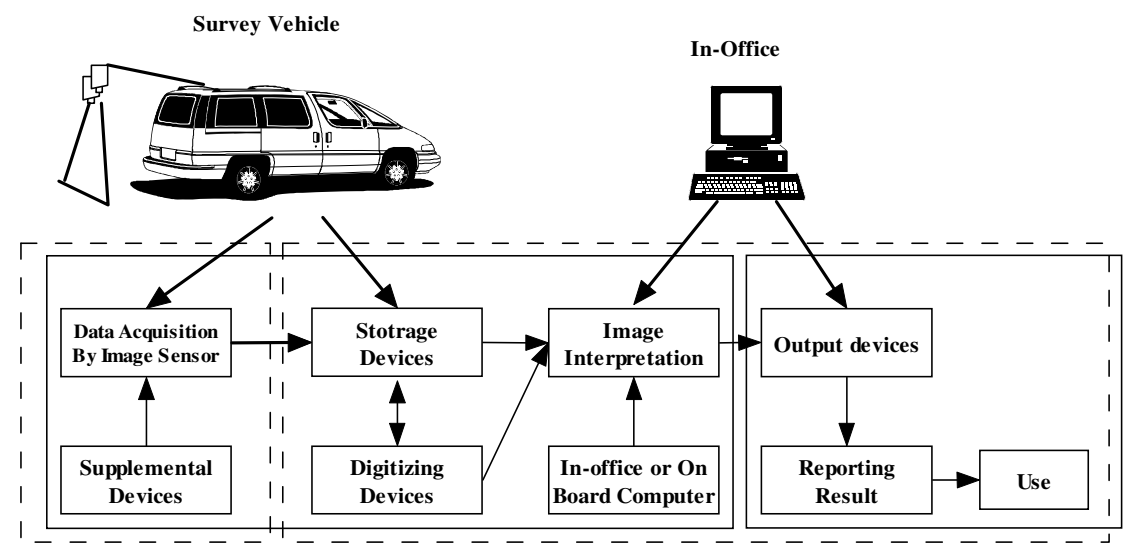
Lengend:
Real-time Processing or On Board Computer Processing
In-office Processing

Fig. 1. Elements of a pavement imaging system 
system is in application at present, the system with surface-scan camera has the problem of low distinguish, and the dynamic collecting image clarity is not very ideal [8]. No method has achieved completely satisfactory results [9].

\section{Significance of Pavement Distress Information}

Pavement distresses are visible imperfections on the surface of pavements. They are symptoms of the deterioration of pavement structures. Agencies that have implemented a Pavement Management System (PMS) collect periodic surface distress in formation on their pavements through distress surveys [10].

Distress information takes a vital role in quantifying the quality of pavements. This information has been used to document present pavement condition, chart past performance history and predict future pavement performance [11]. Pavement distress information is also broadly used as the only quality measure of pavements in many PMS. This is particularly true for systems used by local governments and in urban areas where roughness measurements are not performed because of a lack of equipment availability, high cost or a lack of relative applicability.

\section{Pavement Image Analysis}

The purpose of image processing is to extract the distress features from the pavement image. Preprocessing is done by removing extraneous features that have higher pixel intensities than the mean pixel intensity in the image. In this process, all the pixels representing paint striping and surface textures brighter than the average background gray level are surpassed to the background.

The effect of pavement image processing is illustrated by the following example. This study utilised full programming language software MATLAB 7.10.0 (Mathwork, Natick, MA, USA) to enable a series of MATLAB statements to be written into a file and then execute them with a single command.

\subsection{Image Enhancement}

Image enhancement is applied in an attempt to remove noise in pavement images. Noise reduction is one aspect of preprocessing phase of crack detection process. Filtering is the most common form of noise reduction. Median filtering is one of the most commonly used preprocessing techniques for crack detection today. Median filtering is therefore applied as pavement image enhancement technique as suggested by Jitprasithsiri [12]. The median is much less sensitive than the mean to extreme values. Median filtering is better able to remove these outliers without reducing the sharpness of the image. Such a property of median filtering was explained by a classic example of salt and pepper noise which was a random addition of black and white pixels into a gray scale image. The result of median filtering in figure 6 indicated that noise was removed aptly by the median filtering compared with other operators. 


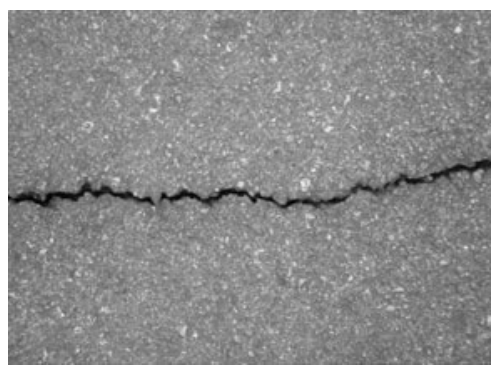

Fig. 2. Original pavement grayscale image

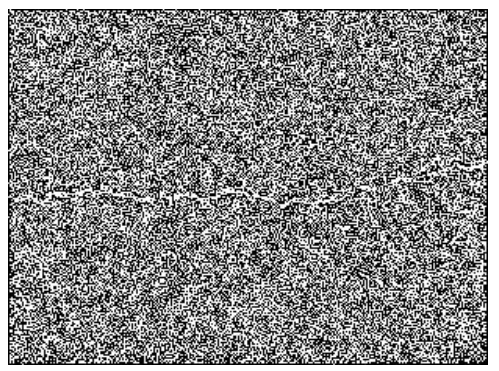

Fig. 4. Laplacian operator

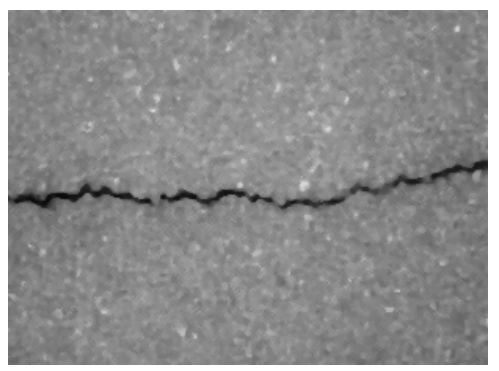

Fig. 6. Result of median filtering

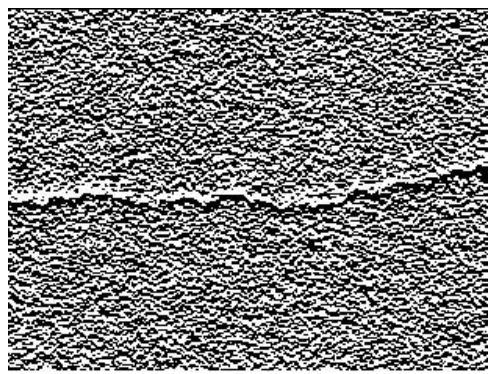

Fig. 8. Sobel operator

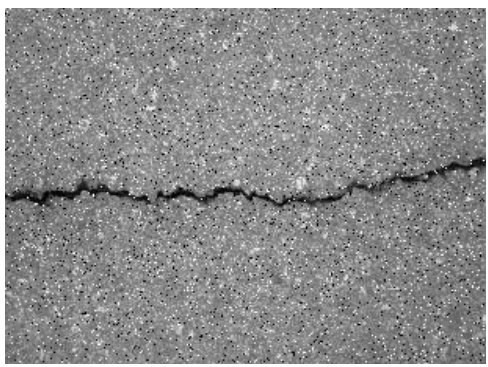

Fig. 3. Grayscale image with salt and pepper

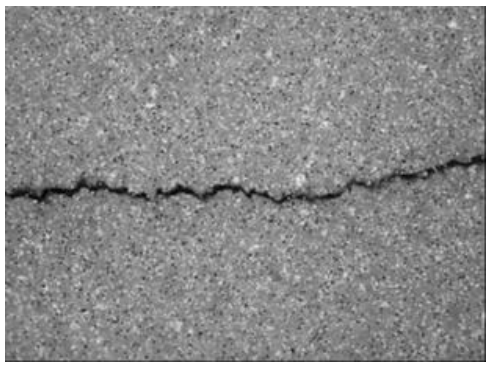

Fig. 5. Gaussian operator

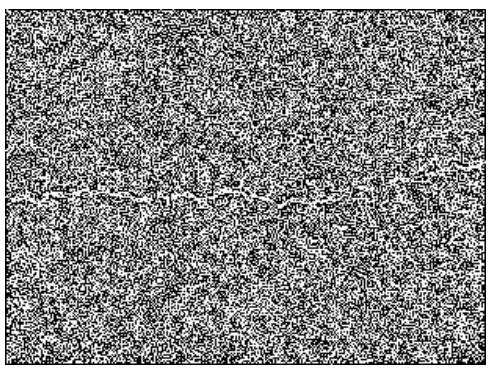

Fig. 7. Log operator

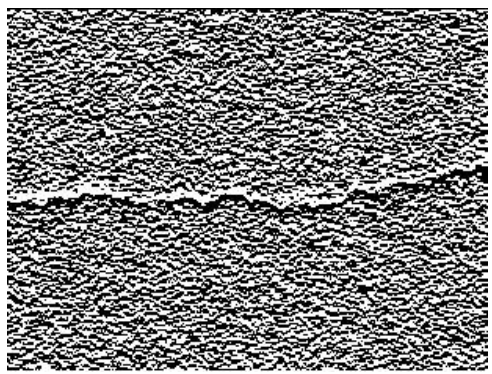

Fig. 9. Prewitt operator 


\subsection{Image Segmentation}

Image segmentation is the crucial step in automatic image distress detection and classification (e.g., types and severities) and has important applications for automatic crack sealing [13]. The segmentation approach chosen was based on a histogram modification technique. This histogram modification was achieved through iterative clipping. At each stage in iterative clipping, more pixels were assigned to the background. This process continues until only distress features were left. The end result as shown in Figure 12 was an image in which the distresses were distinct and easily separable from the background. At this point, a threshold value could be determined automatically in order to isolate the distress features from the background. Transformation function in Figure 11 presented that the threshold value was near 0.6.

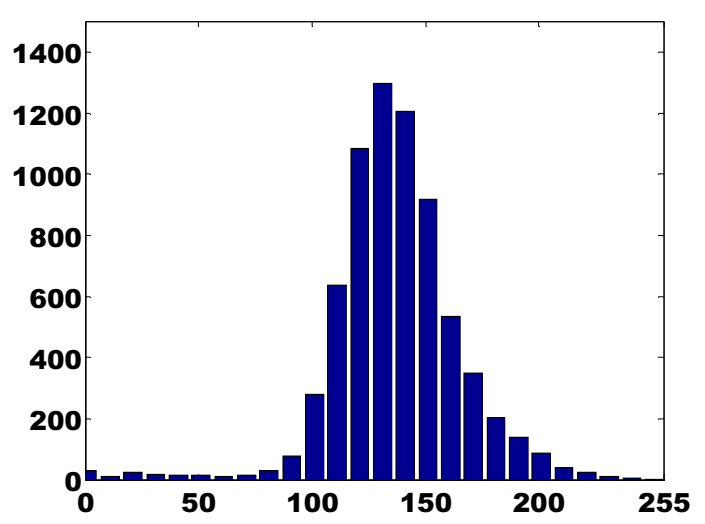

Fig. 10. Histogram of original image

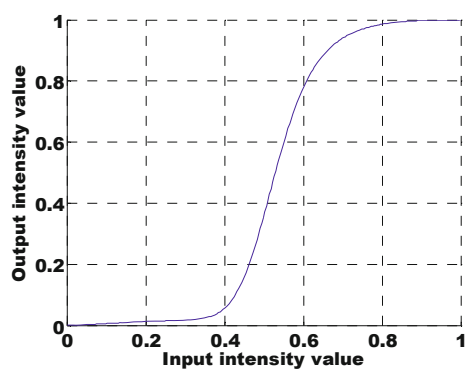

Fig. 11. Transformation function

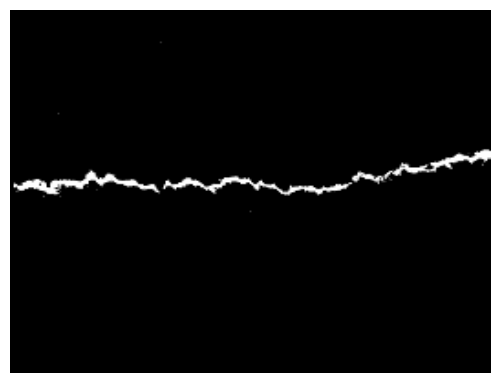

Fig. 12. Result of histogram modification

\subsection{Canny Edge Detection}

The Canny edge detector is a good edge detector among traditional edge detection algorithms [14]. The gray scale pavement image was first smoothed using a Gaussian 
filter with a specified standard deviation to reduce noise (see figure 13), then gradients were calculated to determine edge points. Using two threshold values 0.5 and 0.4 , different correlated edge points were linked together. The strength of the method is its ability to detect edges in the presence of noise and to detect weak edges. Preliminary results from canny edge detection in figure 14 16 showed that the optimum identification of distress was dependent on the parameters used in the algorithm and that the optimum parameters varied with each image. There was a problem of coming up with false distress boundaries when a very high standard deviation in Gaussian filtering was used. The distress may seem wider than it actually was resulting in false severity level detection.

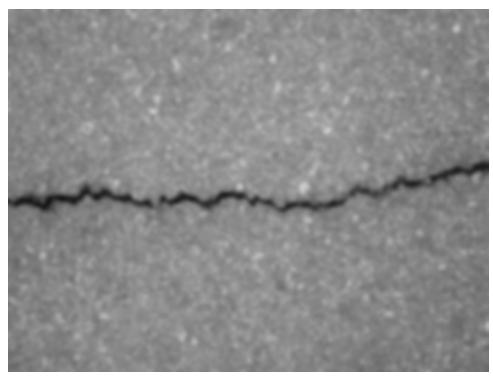

Fig. 13. Grayscale image by gaussian filtering

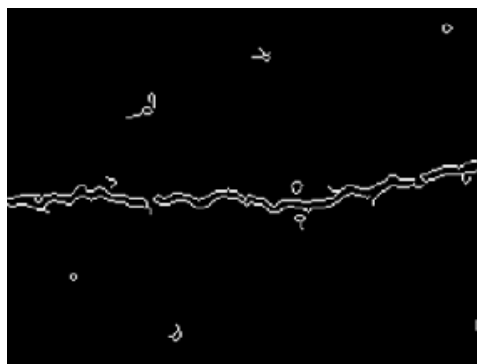

Fig. 15. Threshold value $=0.4$

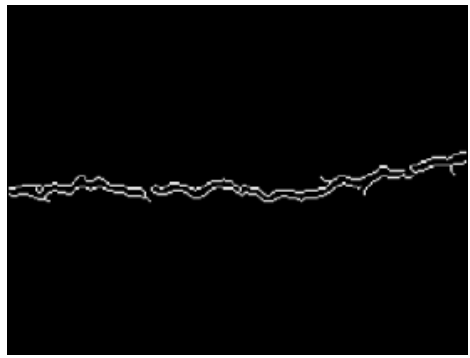

Fig. 14. Threshold value $=0.5$

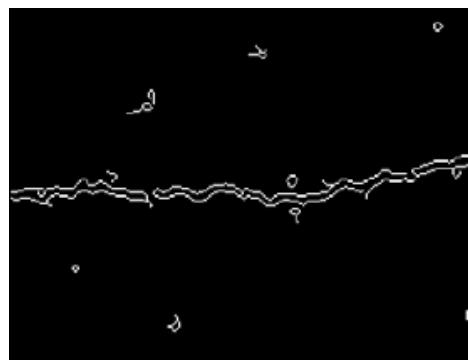

Fig. 16. Result of canny edge detection

\subsection{Pavement Distress Classification and Evaluation}

Many protocols and definitions exist for pavement distresses classification and evaluation. One of the most widely used protocols is Strategic Highway Research Program Long-Term Pavement Performance (SHRP-LTPP) protocol [15]. SHRP-LTPP first classifies the type of cracks according to their orientations, locations, and shapes and quantifies the severity and extent of the cracks according to their properties, such as width, length, and areas. Another important protocol is the World Bank's universal cracking indicator (UCI) [16]. UCI defined a single number to indicate the severity of all the cracks in a pavement segment. For a single crack such as a longitudinal, transverse, or diagonal crack, its indicator was defined as the product of 
its width and length. For the block and alligator cracks, their indicators were defined by the area that contains the block or alligator cracks. The unified crack index (ASTM STP 1121 ) is a protocol similar to UCI. The standard crack density can be automatically determined by dividing the number of pixels for the cracks by the number of the total pixels of the pavement segment.

\section{Conclusions and Perspectives}

As mentioned above, digital images lend themselves to automated analysis because of the ability to analyze variations in grayscale as those variations relate to pavement features. A major force behind the move toward digital imaging of pavements is the opportunity to reduce distress data from those images through automated methods. With the fast development of analog videotaping, using digital imaging to capture pavement surface is becoming the preferred method. With advances in image sensors and computer technologies, the automation of data collection and analysis is a major goal of contemporary pavement management. Several automated analysis algorithms for pavement distress are in use and others are under development.

\section{Acknowledgements}

The authors gratefully acknowledge the financial support provided by National Science and Technology Support Program (2008BAD96B04), Key Laboratory of Ministry of Education for Conveyance and Equipment, East China Jiaotong University (Grant No. 09JD10).

\section{References}

1. Kim, J.: Development of a Low-Cost Video ImagingSystem for Pavement Evaluation. Oregon State University. Ph.D. Thesis (1998)

2. Cheng, H.D., Miyojim, M.: Automatic pavementdistress detection system. Journal of Information Sciences 108, 219-240 (1998)

3. T.R.B.E. NCHRP synthesis 334: Automated Pavement Distress Collection Techniques. Transportation Research Board of the National Academies, Washington, D.C. (2004)

4. Ye, G.R., Zhou, Q.S., Lin, X.W.: Measurement of Surface Crack Width Based on Digital Image Processing. Journal of Highway and Transportation Research and Development 27, 75-78 (2010) (in Chinese)

5. Li, Q.Q., Hu, Q.W.: A Pavement Crack Image Analysis Approach Based on Automatic Image Dodging 27, 1-5 (2010) (in Chinese)

6. Wang, R.B., Wang, C., Chu, X.M.: Developments of Research on Road Pavement Surface Distress Image Recognition. Journal of Jilin University (Engineering and Technology Edition) 32, 91-97 (2002) (in Chinese)

7. Zhang, J.: Study on Pavement Crack Identification and Evaluation Technology Based on digital Image Processing. Chang'an University. Ph.D. Thesis (2004) (in Chinese) 
8. Chu, X.M., Yan, X.P.: The Automatic Search of Pavement Surface Distress Image Based on on-line Learning. In: International Conference on Transportation Engineering 2007 (ICTE 2007), vol. 246, pp. 3282-3287 (2007)

9. Tsail, Y.C., Kaul, V., Russell, M.M.: Critical Assessment of Pavement Distress Segmentation Methods. Journal of Transportation Engineering 136, 11-19 (2010)

10. Haas, R., Hudson, W.R., Zaniewski, J.: Modem Pavement Management. Krieger Publishing Company, Malabar (1994)

11. Shahin, M.Y.: Pavement Management for Airport, Roads and Parking Lots. Chapman \& Hall, New York (1994)

12. Jitprasithsiri: Development of a New Digital Pavement Image Processing Algorithm for Unified Crack Index Computation. University of Utah. Ph.D. Thesis (1997)

13. Mustaffara, M., Lingb, T.C., Puanb, O.C.: Automated Pavement Imaging Grogram (APIP) for Pavement Cracks Classification and Quantification-A Photogrammetric Approach. The International Archives of the Photogrammetry. Remote Sensing and Spatial Information Sciences 37, 362-372 (2008)

14. Canny, J.: A computational approach to edge detection. IEEE Transactions on Pattern Analysis and Machine Intelligence 8, 679-698 (1986)

15. Hawks, N.F., Teng, T.P., Bellinger, W.Y., Rogers, R.B., Baker, C., Brosseau, K.L., Humphrey, L.C.: Distress Identification Manual for the Long-Term Pavement Performance Project, SHRP-P-338. National Research Council, Washington, D.C. (1993)

16. Paterson, W.D.: Proposal of Universal Cracking Indicator for Pavements. Transportation Research Record 1455, TRB, National Research Council, Washington, D.C., pp.69-76 (1994) 